\title{
Extensão, ensino e pesquisa proporcionados pelo projeto "Banco de Dados em Eletrocardiografia Veterinária"
}

\author{
Extension, teaching, and research provided by the "Veterinary Electrocardiography \\ Database project"
}

\author{
Ketlen de Oliveira Benicio ${ }^{1}$ \\ Jaqueline Natalia Pizzaia ${ }^{2}$ \\ Beatriz Kiihl Roque ${ }^{3}$ \\ Deise Carla Almeida Leite Dellova ${ }^{4}$
}

\section{RESUMO}

Este trabalho tem como objetivo compartilhar a experiência e os resultados de um projeto de extensão universitária que possibilita a prática do ensino e pesquisa para os alunos do curso de graduação em Medicina Veterinária. O projeto "Banco de Dados em Eletrocardiografia Veterinária" teve início em 2012, com a finalidade de tornar o exame de eletrocardiograma (ECG) acessível aos pacientes veterinários atendidos na cidade de Pirassununga-SP e região, além de oferecer o treinamento teórico-prático na área de eletrofisiologia cardíaca para os estudantes. Em nove anos, 103 alunos de graduação participaram do projeto e o ECG foi realizado em 1.291 animais de diferentes espécies. Além da avaliação dos animais para o diagnóstico ou exclusão de arritmias cardíacas, os alunos também organizaram eventos destinados à comunidade e desenvolveram projetos de pesquisa. $\mathrm{O}$ armazenamento dos ECG em um banco de dados on-line tem gerado recursos didáticos, a partir de experiências práticas, para diversas disciplinas obrigatórias do curso. $\mathrm{O}$ atendimento especializado aos animais é uma das maneiras de devolver parte do investimento feito pela sociedade na formação dos estudantes de universidades públicas; somado a isso, os resultados deste projeto demonstraram que é possível conciliar as atividades de extensão com ensino e pesquisa.

Palavras-chave: ECG. Animais Domésticos. Práticas Hospitalares. Atendimento Veterinário.

\section{ABSTRACT}

This work aims to share the experience and results of an extension project, which enables the practice of teaching and research for undergraduate students in "Veterinary Medicine. The Veterinary Electrocardiography Database" project started in 2012, with the purpose of making the electrocardiogram (ECG) accessible to veterinary patients treated in the city of Pirassununga, State of São Paulo, and region, in addition to offering theoretical and practical training in cardiac electrophysiology for students. In these nine years, 103 undergraduate students participated in the project and the ECG was performed on 1,291 animals of different species. In addition to the diagnosis or exclusion of cardiac arrhythmias in the animals, the students also organized events for the community and developed research projects. The storage of ECG in an online database generates teaching resources, based on practical

\footnotetext{
${ }^{1}$ Graduanda em Medicina Veterinária na Faculdade de Zootecnia e Engenharia de Alimentos da Universidade de São Paulo, São Paulo, Brasil (ketlenbenicio@usp.br).

${ }^{2}$ Graduanda em Medicina Veterinária na Faculdade de Zootecnia e Engenharia de Alimentos da Universidade de São Paulo, São Paulo, Brasil (jaquelinepizzaia@usp.br).

${ }^{3}$ Mestra em Biociência Animal pela Universidade de São Paulo, São Paulo, Brasil (beatriz.kiihl@gmail.com).

${ }^{4}$ Doutora em Fisiologia pelo Instituto de Ciências Biomédicas da Universidade de São Paulo, São Paulo, Brasil; professora da Faculdade de Zootecnia e Engenharia de Alimentos da Universidade de São Paulo, São Paulo, Brasil (leite-dellova@usp.br).
} 
experiences, for several mandatory subjects of the course. Specialized care for animals is one of the ways to return part of society's investment in the training of students from public universities; in addition, the results of this project demonstrated that it is possible to conciliate extension, teaching and research activities.

Keywords: ECG. Domestic Animals. Hospital Practices. Veterinary Care.

\section{INTRODUÇÃO}

As Diretrizes Curriculares Nacionais ressaltam que a formação do médico veterinário deve ser pautada em atividades práticas para o desenvolvimento de competências e habilidades na área médica voltada para os animais (BRASIL, 2019). Portanto, os alunos do curso de graduação em Medicina Veterinária exercem atividades práticas no hospital veterinário, que representam uma parte importante da formação acadêmica. Mas, além de serem fundamentais, essas atividades permitem aos alunos o exercício da extensão universitária, como por exemplo, o atendimento clínico aos animais de uma cidade ou região.

A Unidade Didática Clínico-Hospitalar (UDCH) da Faculdade de Zootecnia e Engenharia de Alimentos da Universidade de São Paulo (FZEA/USP) foi idealizada e estruturada para proporcionar o atendimento clínico e cirúrgico para os animais de interesse zootécnico e de companhia de Pirassununga-SP e região. Além disso, a rotina de atendimentos da UDCH permite o desenvolvimento de projetos de ensino e extensão.

Neste relato, pretende-se compartilhar a experiência e os resultados de um projeto de extensão na área de eletrocardiografia que também possibilita a prática do ensino e da pesquisa para os alunos do curso de graduação em Medicina Veterinária.

\section{Eletrocardiografia}

A eletrocardiografia é um método não invasivo adotado para o registro da atividade elétrica do coração, utiliza-se um aparelho denominado eletrocardiógrafo e eletrodos fixados à pele. Isso é possível porque a atividade elétrica cardíaca se propaga em diferentes regiões e alcança a superfície corporal (BRUNNER; SUDDARTH; LIPPINCOTT, 2011).

O eletrocardiógrafo é um galvanômetro que capta o potencial elétrico cardíaco, convertendo-o num registro gráfico da amplitude em função do tempo, denominado de eletrocardiograma (ECG) (SANTILLI et al., 2018). No ECG são observadas ondas específicas, que representam as fases de despolarização (ondas $\mathrm{P}, \mathrm{Q}, \mathrm{R}$ e S) e repolarização 
(onda T) do miocárdio e que resultam na contração e relaxamento das câmaras cardíacas (átrios e ventrículos) (MILL, 2018).

O ECG é um exame complementar de baixo custo e o seu registro não gera dor ou desconforto intencionais para o animal, sendo muito utilizado na Medicina Veterinária para o diagnóstico de arritmias e distúrbios de condução do impulso elétrico no coração (SANTILLI et al., 2018).

Os acadêmicos aprendem os conceitos básicos de eletrocardiografia no início do curso, na disciplina de Fisiologia Animal, e aplicam esses conhecimentos mais adiante, nas disciplinas de Anestesiologia, Clínica Médica e Clínica Cirúrgica. Além disso, saber realizar e interpretar o ECG acaba sendo um diferencial na atividade do médico veterinário.

Um dos primeiros estudos em eletrocardiografia foi publicado em 1888, a partir de observações feitas em cães, e desde então, muitos avanços foram conquistados em relação à metodologia e aos equipamentos (WALLER, 1888; MARGARIDO et al., 2019). Entretanto, o ECG não é amplamente solicitado para os pacientes veterinários pela falta do especialista ou por restrições financeiras dos tutores, que precisam distribuir os recursos entre alimentação, higiene e atendimento veterinário para os animais (SPC BRASIL; CNDL, 2017).

\section{Projeto de extensão na área de Eletrocardiografia Veterinária}

O projeto Banco de Dados em Eletrocardiografia Veterinária teve início há 9 anos na FZEA/USP, com a finalidade de tornar o ECG acessível aos pacientes veterinários atendidos na cidade de Pirassununga/SP e região, além de oferecer o treinamento teórico-prático na área de eletrofisiologia cardíaca para os estudantes de Medicina Veterinária. Para isso, a carga horária de atividades dos estudantes no projeto era de 40 horas mensais e o treinamento prático era realizado diretamente nos animais. De acordo com o Conselho Nacional de Controle de Experimentação Animal (CONCEA), o uso de animais em atividades didáticas é permitido quando se pretende desenvolver habilidades psicomotoras e competências nos discentes envolvidos e para a realização de abordagens diagnósticas, terapêuticas e profiláticas, priorizando, assim, a saúde e a qualidade de vida dos pacientes veterinários (BRASIL, 2021).

Outra atividade do projeto foi a criação e manutenção de um banco de dados a partir do armazenamento digital do ECG, que se tornou uma fonte de pesquisa para os alunos e docentes de várias disciplinas do curso de Medicina Veterinária da FZEA/USP. Além disso, atualmente as informações são disponibilizadas on-line, o que facilita a consulta no âmbito 
científico e permite o uso dos dados em estudos retrospectivos e observacionais. O banco de dados contém o registro eletrocardiográfico gerado e as informações do animal, tais como a idade, raça, peso corporal e estado de saúde. Entretanto, o acesso é limitado aos usuários cadastrados, sendo que a realização do exame e o armazenamento das informações foram autorizados pelo tutor do animal, mediante a assinatura de um termo de consentimento livre e esclarecido. O projeto também foi aprovado pela Comissão de Ética no Uso de Animais da FZEA/USP e protocolado sob o CEUA n ${ }^{\circ} 6212030320$.

Ademais, é importante salientar o serviço prestado à comunidade de Pirassununga-SP e região por meio da avaliação de diferentes espécies como: cães, gatos, equinos e ruminantes. Nos animais, principalmente nos cães e gatos, as arritmias podem ser assintomáticas por longos períodos e, dessa forma, a avaliação eletrocardiográfica periódica acaba sendo imprescindível, ainda mais quando se pretende realizar o diagnóstico precoce das doenças cardíacas (MEURS, 2017).

No início, os alunos inscritos no projeto foram treinados para a realização do exame físico, com maior atenção para o sistema cardiovascular, e o registro do ECG nas diferentes espécies animais. Vale ressaltar que além da manipulação do eletrocardiógrafo, os alunos precisam saber como conter e posicionar adequadamente os pacientes. Por exemplo, cães e gatos são contidos, gentilmente, em decúbito lateral, enquanto os bovinos e equinos realizam o exame em estação (Figura 1). Os animais domésticos, como cães, gatos, bovinos, caprinos, ovinos e equinos realizam o ECG acordados, ou seja, sem a administração de sedativos ou anestésicos, diferente do que ocorre nos animais silvestres (MBITHI; MULEI; MOGOA, 2003).

Concomitantemente, os alunos receberam o treinamento para a interpretação do ECG e elaboração do laudo, comparando os resultados do exame com as informações da literatura científica especializada (LESSA; ESCODRO; NOTOMI, 2012; OLIVEIRA et al., 2008; SANTILLI et al, 2018). O treinamento foi fornecido por docentes e médicos veterinários capacitados, que depois passaram a supervisionar as atividades dos estudantes. 
Figura 1 - Exemplos de posicionamento de animais para realização do eletrocardiograma

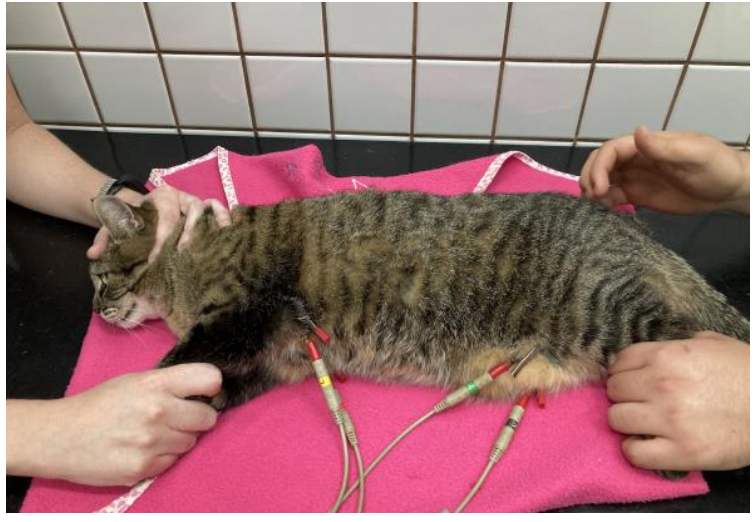

A) Gata, sem raça definida, posicionada em decúbito lateral e com os eletrodos fixados à pele dos membros torácicos e pélvicos.

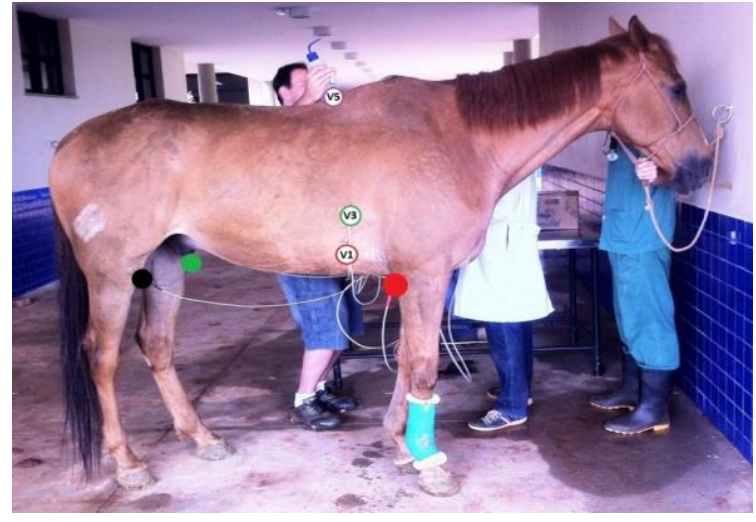

B) Equino, sem raça definida, em estação e com os eletrodos fixados à pele dos membros torácicos e pélvicos e do tórax.

Fonte: Os autores (2021).

Nesse projeto, além do treinamento teórico-prático na área de eletrofisiologia cardíaca e do atendimento aos pacientes, os alunos precisaram desenvolver habilidades de comunicação e interação com os tutores para a obtenção de informações sobre o estado de saúde do animal e prescrição dos tratamentos indicados. Ao mesmo tempo, os tutores criam expectativas em relação ao atendimento, buscando conhecimento técnico, atenção, alternativas terapêuticas e o manejo adequado dos seus animais por parte dos estudantes e Médicos Veterinários (NIEDERAUER, 2015). E, certamente, a experiência e a competência das várias equipes que se formaram ao longo destes anos de projeto supriram essas expectativas.

\section{Desdobramentos do projeto}

Nesses nove anos, 103 alunos de graduação participaram do projeto "Banco de Dados em Eletrocardiografia Veterinária”, entre bolsistas e voluntários. Até julho de 2021, o ECG foi realizado em 1.291 animais, sendo: 1.160 cães, 32 gatos, 80 equinos, 16 ruminantes (14 bovinos, 1 caprino e 1 ovino), 1 ganso (Anser anser), 1 jaguatirica (Leopardus pardalis) e 1 macaco prego (Cebus nigritus).

As arritmias são comuns em cães, como resultado de alterações diretas no sistema de condução cardíaca ou em decorrência de complicações relacionadas a outras doenças, como a doença renal e endócrina (NELSON; COUTO, 2015). Portanto, na rotina hospitalar, o ECG é mais frequentemente realizado em cães do que em outras espécies. Os cães sem raça definida 
são os mais atendidos na rotina; entretanto, cães de determinadas raças, como Boxer, Golden Retriever, Pinscher e Dachshund estão entre os que mais apresentam arritmias cardíacas (MORAES; CAETANO; LEITE-DELLOVA, 2020).

Os laudos dos ECG foram disponibilizados aos médicos veterinários e tutores sem nenhum custo. Os principais motivos para a realização do ECG foram a avaliação préoperatória ou de rotina em animais idosos, a suspeita de arritmias cardíacas e o acompanhamento clínico de animais com cardiopatias.

Ao longo destes anos, alterações eletrocardiográficas importantes foram observadas principalmente em cães com oito anos de idade ou mais (SILVA et al., 2018). Entre elas, batimentos ectópicos (atriais e ventriculares) e distúrbios de condução elétrica (bloqueios atrioventriculares e bloqueios de ramo), que prejudicam a atividade normal do coração (MORAES; CAETANO; LEITE-DELLOVA, 2020).

Desde o início do projeto, nove projetos de pesquisa foram desenvolvidos em conjunto com as atividades de extensão, e os resultados obtidos nesses projetos foram apresentados em eventos de iniciação científica e relacionados à área de clínica médica veterinária, além de terem sido publicados na forma de artigos científicos (BOMFIM et al., 2017; MARGARIDO et al., 2017; 2019; SILVA et al., 2018; MORAES; CAETANO; LEITE-DELLOVA, 2020). Portanto, o projeto "Banco de Dados em Eletrofisiologia Veterinária" gerou produção científica, demonstrando que também é possível conciliar as atividades de extensão e pesquisa.

Os alunos envolvidos com o projeto também participaram da organização de eventos destinados à comunidade, como a I Cãominhada, que ocorreu em 21 de maio de 2016, e a II Cãominhada, que ocorreu em 27 de maio de 2017 (Figura 2). Esses dois eventos tiveram a finalidade de estimular a atividade física conjunta entre os tutores e seus respectivos cães, por meio de uma caminhada de aproximadamente $2 \mathrm{~km}$ no Campus Fernando Costa em Pirassununga-SP. A I e a II Cãominhada contaram com 450 participantes, entre voluntários e moradores de Pirassununga, e 190 cães. Antes da atividade física, os cães foram submetidos a uma avaliação clínica geral, a fim de identificar possíveis contraindicações ao exercício. As avaliações foram realizadas pelos alunos do $7^{\circ}$ e $9^{\circ}$ semestres do curso de Medicina Veterinária da FZEA/USP. Os alunos do $3^{\circ}$ ao $9^{\circ}$ semestres também interagiram com o público, conscientizando os tutores sobre a relevância de consultas periódicas e chamando a atenção deles para as manifestações clínicas relacionadas às doenças cardíacas em cães. 
Figura 2 - I e II Cãominhada realizadas no campus Fernando Costa em Pirassununga-SP

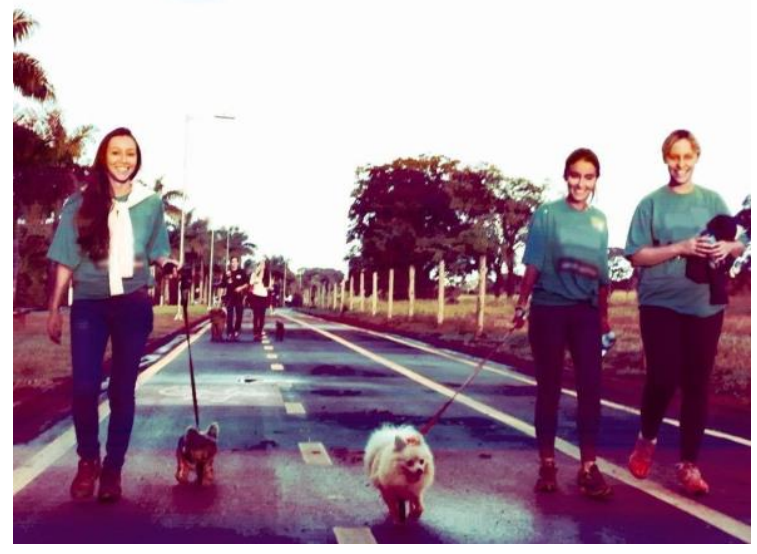

A) Alunos, tutores e seus respectivos cães realizando atividade física durante a I Cãominhada em 2016.

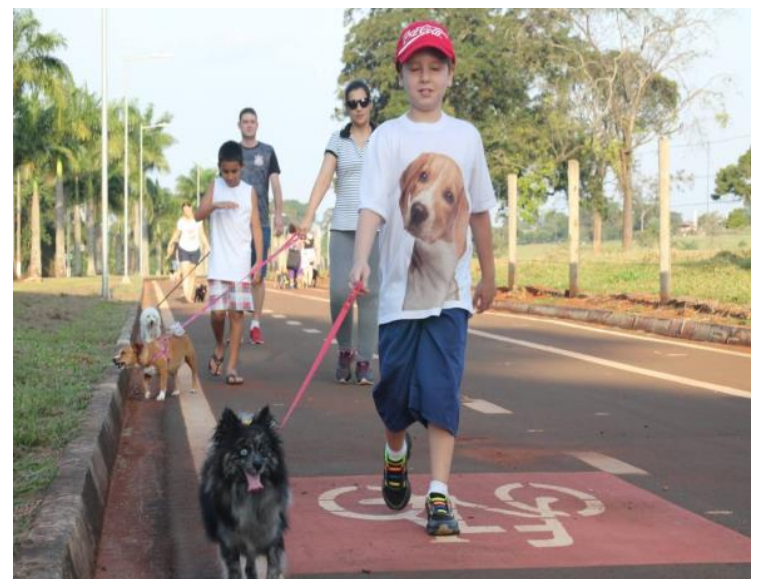

B) Tutores e seus respectivos cães realizando atividade física durante a II Cãominhada em 2017.

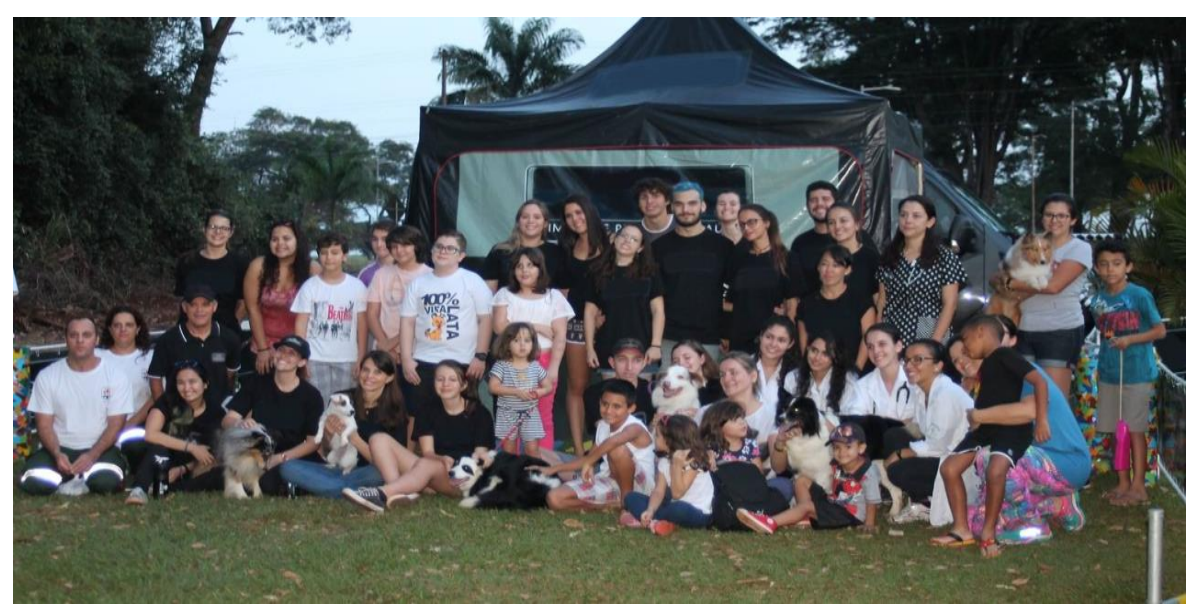

C) Voluntários e participantes da II Cãominhada em 2017.

Fonte: Reprodução das redes sociais (2021).

\section{CONSIDERAÇÕES FINAIS}

Desde o início do projeto em 2012, 1.291 animais foram avaliados por meio do ECG, gerando aprendizado na prática para os alunos de graduação em Medicina Veterinária da FZEA/USP. Os alunos participaram diretamente das avaliações e ampliaram os conhecimentos nessa especialidade, que é pouco abordada durante a formação. Também foram gerados recursos importantes e únicos para disciplinas obrigatórias, além de um atendimento diferenciado para a comunidade de Pirassununga-SP e região. Dessa forma, criou-se um ambiente de troca, no qual o paciente veterinário tem acesso a uma avaliação 
clínica gratuita e de qualidade, e os alunos têm a oportunidade de aprimorar seus conhecimentos e acompanhar a rotina hospitalar.

Além disso, a participação dos alunos em eventos abertos à comunidade gerou resultados positivos, uma vez que eles compartilharam informações de uma forma mais acessível ao público e divulgaram atividades realizadas na UDCH da FZEA/USP, entre elas o projeto "Banco de Dados em Eletrofisiologia Veterinária".

\section{AGRADECIMENTOS}

Agradecemos ao Departamento de Medicina Veterinária e à Diretoria da FZEA/USP e à Prefeitura do Campus Fernando Costa em Pirassununga pelo apoio às atividades deste projeto. Agradecemos também à Universidade de São Paulo e às agências de fomento (Fundação de Apoio à Pesquisa do Estado de São Paulo - FAPESP - processos: 2012/240728, 2014/27085-9, 2014/27242-7 e 2015/17876-1, e Conselho Nacional de Desenvolvimento Científico e Tecnológico - CNPq - processos: 101815/2016-6, 117391/2017-4 e 148655/2021-1) pelas bolsas de estudo, aos tutores dos animais e aos médicos veterinários que participaram dos atendimentos.

\section{REFERÊNCIAS}

BOMFIM, M. M. et al. Physical and electrocardiographic evaluation of horses used for wagon traction. Arquivo Brasileiro de Medicina Veterinária e Zootecnia, Belo Horizonte, v. 69. n. 2, p. 371-376, 2017. Doi: 10.1590/1678-4162-9248. Disponível em: https://www.scielo.br/j/abmvz/a/gHtM6TgDZ9pWRXx8dTpN4wC/?lang=en. Acesso em: 07 set. 2021.

BRASIL. Ministério da Ciência, Tecnologia e Inovações/Conselho Nacional de Controle de Experimentação Animal. Resolução no 53, de 19 de maio de 2021. Dispõe sobre restrições ao uso de animais em ensino, em complemento à Diretriz Brasileira para o Cuidado e a Utilização de Animais em Atividades de Ensino ou de Pesquisa Científica - DBCA. Disponível em: https://www.in.gov.br/en/web/dou/-/resolucao-normativa-n-53-de-19-demaio-de-2021-321569251. Acesso em: 22 dez. 2021.

BRASIL. Ministério da Educação. Conselho Nacional de Educação. Câmara de Educação Superior. Resolução $\mathbf{n}^{0}$ 3, de 15 de agosto de 2019. Institui as Diretrizes Curriculares Nacionais do Curso de Graduação em Medicina Veterinária e dá outras providências. Disponível em: https://www.in.gov.br/en/web/dou/-/resolucao-n-3-de-15-de-agosto-de-2019210946881. Acesso em: 14 out. 2021.

BRUNNER, L. S.; SUDDARTH, D. S.; LIPPINCOTT, W. W. Brunner \& Suddarth: Exames Complementares. Rio de Janeiro: Grupo GEN, 2011. p. 162-164. 
LESSA, D. F. S.; ESCODRO, P. B.; NOTOMI, M. K. Considerações sobre o uso da eletrocardiografia e sua interpretação em equinos. + Equina, Jacareí, v. 42, p. 4-12, 2012.

MARGARIDO, M. P. et al. Evaluation of electrocardiographic parameters, thoracic morphometry and vertebral heart size in clinically normal dogs. Acta Scientiae Veterinariae, Porto Alegre, v. 47, n. 1, 2019. Doi: 10.22456/1679-9216.98194. Disponível em: https://seer.ufrgs.br/ActaScientiaeVeterinariae/article/view/98194/55262. Acesso em: 2 out. 2021.

MARGARIDO, M. P. et al. Relação entre as dimensões do coração, a morfometria torácica e as ondas do ECG em cães. In: CONGRESSO BRASILEIRO DA ANCLIVEPA, 38., 2017, Recife. Anais [...]. Pernambuco: ANCLIVEPA, 2017.

MBITHI, P. M. F.; MULEI, C. M.; MOGOA, E. G. M. Restraint of domestic, laboratory and wild animals: a manual for veterinary students, practitioners and animal handlers. Nairobi: University of Nairobi Press, 2003. p. 95-102.

MEURS, K. M. Arrhythmogenic right ventricular cardiomyopathy in the boxer dog: an update. Veterinary Clinics of North America: Small Animal Practice, [s. l.], v. 47, n. 5, p. 1103-1111, 2017. Doi: 10.1016/j.cvsm.2017.04.007. Disponível em: https://www.sciencedirect.com/science/article/pii/S0195561617300402?via=ihub. Acesso em: 10 out. 2021.

MILL, J. G. Bases fisiológicas da eletrocardiografia. In: AIRES, M. M. Fisiologia. 5. ed. Rio de Janeiro: Grupo GEN, 2018. p. 95-102.

MORAES, P. de; CAETANO, Y. C.; LEITE-DELLOVA, D. C. A. Arritmias em cães com raça definida. In: SIMPÓSIO INTERNACIONAL DE INICIAÇÃO CIENTÍFICA E TECNOLÓGICA DA UNIVERSIDADE DE SÃO PAULO, 28., 2020, Pirassununga. Anais [...]. São Paulo: USP, 2020. Disponível em: https://uspdigital.usp.br/siicusp/siicPublicacao.jsp?codmnu=7210. Acesso em: 26 set. 2021.

NELSON, R. W.; COUTO, C. G. Arritmias cardíacas e terapia antiarrítmica. In: NELSON, R. W. (org.). Medicina interna de pequenos animais. 5. ed. Rio de Janeiro: Grupo GEN, 2015. p. 73-95.

NIEDERAUER, A. L. O. Estudo sobre as percepções na relação médico veterinário, tutor e estudante de medicina veterinária em relação à clínica veterinária de pequenos animais no hospital de clínicas veterinárias da UFRGS. 2015. 58 f., Monografia (Graduação em Medicina Veterinária) - Universidade Federal de Porto Alegre, Porto Alegre, 2015. Disponível em: https://lume.ufrgs.br/handle/10183/207560. Acesso em: 15 out. 2021.

OLIVEIRA, P. C. L. de et al. Padronização da técnica de execução e parâmetros eletrocardiográficos normais, em derivações periféricas, para bovinos indianos adultos (Bos taurus indicus) da raça Nelore. Ceres, Viçosa, v. 55, n. 3, p. 224-230, 2008. Disponível em: http://www.ceres.ufv.br/ojs/index.php/ceres/article/view/3314/1199. Acesso em: 26 set. 2021.

SANTILLI, R. et al. Electrocardiography of the dog and cat: diagnosis of arrhythmias. 2. ed. Milano: Edra, 2018.

SILVA, T. V. S. de et al. Alterações eletrocardiográficas em cães da rotina hospitalar. In: SIMPÓSIO INTERNACIONAL DE INICIAÇÃO CIENTÍFICA E TECNOLÓGICA DA 
UNIVERSIDADE DE SÃO PAULO, 26., 2018, Pirassununga. Anais [...]. São Paulo: USP, 2018. Disponível em:

SPC BRASIL. $61 \%$ dos donos de animais de estimação veem seus pets como um membro da família; gasto mensal é de $\mathbf{R} \$ \mathbf{1 8 9}$, em média. 2017. Disponível em:

https://www.spcbrasil.org.br/pesquisas/pesquisa/3540. Acesso em: 16 out. 2021.

WALLER, A. D. Introductory address on the electromotive properties of the human heart. British Medical Journal, Bethesda, MD, v. 2, n. 1.449, 1888. Doi:

10.1136/bmj.2.1449.751. Disponível em:

https://www.ncbi.nlm.nih.gov/pmc/articles/PMC2198454/. Acesso em: 16 out. 2021.

Submetido em 21 de outubro de 2021.

Aprovado em 13 de dezembro de 2021. 